\title{
OPTIMAL KEY GENERATION FOR PRIVACY PRESERVATION USING BLOCKCHAIN TECHNOLOGY
}

\author{
${ }^{1}$ Priti Lale \\ Research Scholar, Computer Science \& Engineering, \\ Suresh Gyan Vihar University Jaipur, India \\ priti.met@gmail.com \\ ${ }^{2}$ Mukesh Kumar Gupta \\ Professor, Electrical Engineering, \\ Suresh Gyan Vihar University Jaipur, India \\ mukeshkr.gupta@mygyanvihar.com \\ ${ }^{3}$ Manish Sharma \\ Professor, Computer Science \&Engineering, \\ Jaipur National University, Jaipur, India \\ manish.sharma@mygyanvihar.com
}

\begin{abstract}
Supply chain management is an important area of logistics. It is a process involving many stakeholders with multiple areas and even more moving parts. The proposed technology aims to make the supply chain more efficient, investing in the wrong technology complicates productivity further while hampering profits, leading to multiple issues in supply chain. Blockchain technology is the solution to the problem of structuring data with built-in security features. Blockchain technology enables decentralization with the participation of members on distributed networks. There is not a single point of failure because of the distributed structure and the transaction record cannot be change by single user. In this paper for making data more secure while sharing information in supply chain a key generation process is done using data sanitization and restoration process and new improved Fittest Self Adaptive sea lion algorithm is discussed using only the authorize person will be able to use shared data and it will be compared with existing model using different parameters.
\end{abstract}

Keywords: Blockchain Technology; Supply chain management; security; data sanitization; data restoration.

\section{Introduction}

The traditional supply chain is difficult to fulfil the demand of customers asking for high quality with reasonable price. Integrating the current trend and making the existing system smart is need of the automation and sustainability. Integration of communication technology and information with the supply chain is the area where researchers are working on and are constantly striving to achieve efficient Supply chain [1]. In recent times, the price war and the high demand for quality have led to conflicts between manufacturing organizations and the service sector. For achieving high quality and low cost customer support is needed for new technology integration in the system. It has often been found that consumers face many problems such as late delivery without prior notice, poor quality product, incorrect product due to human negligence, product broken or damaged during delivery, expensive and painful delay [2-4].

Supply chain refers to the flow of products and related information that is exchanged between companies. The direction of this flow goes from supplier to customer and there is a complex exchange and conversion between the origin and distribution of the product. Thus, supply chain management is essential in supply chain coordination. The supply chain faces many challenges: product discovery and capacity, inventory management, quality control and follow-up schedules. Delays affecting a company's finances, growth, and reputation are common. This is exacerbated by the fact that the required information is not always accurate or available, a consequence of which is the lack of manual processing and reliable information technology to incorporate information into the systems of some companies that can collect information. In a safe and fast way. One way to approach these problems of supply chains is to prioritize them and update the supporting technologies. There are multiple systems in use for data exchange for Supply Chain Management such as EDI (Electronic Data Exchange),POS (Point of Sales), VMI (Vendor Managed inventory) and VAN (Value Added Network) .These 
systems work only on systematization of current applications and the optimization for individual applications within the company.

Nowadays, in order to cope with global competition, organizations need to better manage their supply chain; therefore, Organizations are committed to improvement in managing that supply chain. The supply chain covers all related activities making goods from raw materials to deliver final products is information and financial pursuit where resources are both in the process. The pharmaceutical sector has an important role to play in the health and medical system. In most countries, due to the unique type of drug supply and demand the drug market is not regulated regularly [5, 6].Depends on it Competition in the drug market governments need to regulate the financial and clinical side. Drug supply chain guarantees the availability of the right drugs.

The availability of supply chain is a big challenge, unless it becomes simple and effective on a case on case basis cooperation between customer needs and demands supply chain partners is the main way to achieve competitiveness benefits and information sharing among these members. One aspect of the supply chain is information sharing. Various studies have proven the positive effect of information exchange reducing costs so that supply chain management improves. One of the main advantages is multiple parties will involve in it [7-11]. As a result, the supply chain will give better performance in terms of financial returns, level of services and working hours. Information shared between manufacturers and retailers, manufacturers can use retailer inventory level information for time management financial pressures, trade disputes, and global health crises are perceived problems that can seriously worsen.

Supply chain loopholes can add a risk that will increase over time, causing a downturn or even collapse supply chain ecosystem. Therefore, there must be a protocol in place for such situations. If suppliers, customers and logistics are spread all over the world, it needs to be carefully managed and coordinated regulatory differences, tax changes, different time zones and sometimes cultural characteristics. There are a different factor which has a major role to play in improving performance and reducing cost [23-26].

1) Location: Deciding on the location and storage facilities of factories is never easy. The decisions on location determine the time required for the market and, ultimately, the market share and sales.

2) Production: Production planning is a frequent and adult problem and is a complete function of demand forecasting.

3) Inventory: Companies spend $40 \%$ of the total value on inventory management, thus creating the necessary variables in the supply chain optimization equation.

Planning data is critical in supply chain. Successful operational plans and projections are a function of data which is accurate and relevant. In supply chain optimization the inability of accessing relevant data and quality is crucial. It also brings into account cleaning, data enrichment, costs related to data collection, access, storage and security, thus adding multiple dimensions to the data challenge. Ultimately information sharing is a crucial part while maintaining security of data [12-14]. Over the past twenty years, the supply chains of retailers and manufacturer have become even difficult. In many industries, retail orders trigger manufacturers to replenish. Manufacturers with sluggish-tune-in-time supply chains may automatically shut down retail shelves as they sell products. As cooperation increased, companies can use advanced analytics tools to improve results using additional data from partners. Response systems identify them before potential problems arise. Traditional response orders are reduced when a customer demands more product than the manufacturer's delivery. This ensures that the buyer does not matter and that the manufacturer's service is poor. Producers who expect scarcity before the buyer is disappointed can offer alternative products or other incentives to keep the buyer happy. There are many techniques available for the transactions and to manage the large amount of data. But as the all organization have the different protocols regarding transactions ,the involvement of many people in the chain for all the process it takes lot of time and the security issues arise in the traditional systems. Also, by allowing the information sharing and involvement of the consumers this will increase the transparency between the suppliers and the consumers. According to the system created this will increase the trade and economy of the market. As this system is using the block chain technology this will create a decentralized system so that the anti-conflict will also be reduced simultaneously it will help to detect the frauds and avoid it as soon as possible.

In dynamically optimizing the price, shelf life is limited in seasonal products, especially fashion products. Any items not sold at the end of the season are sold at scrap to empty the warehouse with deep discounts. Hotels, airlines and other companies with limited but perishable products dynamically adjust prices to meet demand. This is more difficult with other products such as clothing, where supply can vary greatly; the same forecasting technique can improve margins. Whenever the role of supply chain comes into the play the different aspect of it need to be considered as it operates on different domains and everywhere multiple parties are involve in the communication and if loopholes arise then it will be difficult to identify and make it correct. 


\section{Related Work}

Blockchain technology can contribute to tracking orders, receipts and payments by recording the flow of each asset on the supply chain, while effectively contributing to the integrated and transparent tracking of digital assets such as warrants and licenses. It provides an analysis of blockchain fit in detail in the supply chain industry through its methodology. It defines specific components of a blockchain that affect the supply chain such as efficiency, scalability, privacy considerations, consensus mechanism, location proofs and details of what will affect the blockchain in disrupting the price and supply chain industry. This concludes as a result of a suggested high-level architectural approach to trade-off discussions between consensus pricing, throughput and validity periods, and discussions on the challenges facing the deployment of blockchain in the supply chain industry. The technical features of modern blockchain can effectively facilitate cases where the supply chain is used, but various other challenges bring before us the changes and further research efforts required to achieve a global, product-level blockchain for the supply chain industry. Blockchain technology has been used successfully in many industries such as energy and finance. For the supply chain, the solution has been proposed theoretically without significant consequences in reality situations. Analyse their use of thoughtful technology for blockchain in the field of logistics and supply chain management and their thinking based on the principles of technology adoption with five ideologies: engaging with technology; Looking for technological innovation; Spatial context awareness; Information on alternative technologies; And expect changes in technology[15-18].

Blockchain, basically blockchain, is a growing list of blocks also called as records, using cryptography these are connected. Each block contains the hash of the previous block, transaction data and a timestamp (usually shown as the Merkel tree). By design, blockchain is resistant to data improvement. It is an open, distributed account that can verifiably, effectively and permanently record transactions between two involved parties. For use as a distributed account, blockchain typically follow a protocol for inter-node communication and verification of new blocks through a peer-to-peer network. Once recorded, the data in a given block cannot be preceded by a change in all subsequent blocks, which requires a network majority consensus. Although blockchain records are not irreversible, blockchain can be exemplify distributed computer systems with high Byzantine fault tolerance and by design secured. Blockchain has been claimed as a decentralized consensus. The block contains batches of hashed and encoded valid transactions in Merkel's tree. Each block has a header with information about its block and some information about the previous block. Part of that information is the hash of the title of the previous block. The output is completely different if there is some change in the input of the hash function. Each block contains the cryptographic hash of the previous block in the blockchain, to which two are added. Connected blocks form a chain. This iteration process confirms the integrity of the previous original block, returning to the original origin block.

Etherium and smart contracts are two major areas of it. For basic smart contracts bitcoin was the first supporter in the area where the network could transfer value between two different people. Network compromises of different nodes will only validate a transaction if specific conditions are met. However, Bitcoin is limited in terms of currency usage. Etherium replaces the more restrictive language of Bitcoin (the scripting language) and instead replaces which permits developers to write their own programs with different language. The Etherium White Paper allows Etherium developers to program their own smart contracts or "autonomous agents." The language is 'touring-complete', i.e. it supports a wide set of computer instructions.

Most companies do not need to share information, as they do not want to share their potential with competitors. It is also important to consider access control on the information sharing plan. It is difficult to obtain and describe status updates from a broad perspective and to evaluate performance and constraints. We consider low cost and accessible database systems to meet these requirements. Companies reap the benefits of sharing information through blockchain, but the company's concern about the protection of order content is growing. So, the requirements are likely to be met.

There are researchers who discussed and developed different approach using blockchain technology for security in different domain. In SCMIS approach which is Supply Chain Management and Information Systems it talked about mass customization, Mass customization is the process of services and delivering goods in a market that is modified to suit the needs of a particular customer. Mass customization is a production and marketing technique that combines personalization and flexibility of customize products with low unit costs associated with large-scale production. In blockchain technologies the major benefits identified by researcher is because of the nature of decentralization it is very effective in handling each node in the communication as an independent one and the problems of dependency will not be there. Blockchain technology is used in multiple SCM domains with applications ranging from airlines systems to healthcare, and this section outlines the different methods identified by researchers in different applications. Crowdsourcing systems make use of the human intelligence for solving complex tasks and have gained noticeable interest and adoption in recent years. However the majority of crowdsourcing systems rely on centralized system using central servers, which are subject to the bottleneck for traditional trust-based model, such as single point of failure. They are also 
vulnerable to attacks such as DDOS. The system may conceptualize a blockchain based decentralized framework for crowdsourcing named CrowdBC, in which crowd of workers solve requesters request without relying on any third party, user's privacy can be guaranteed. The extensive involvement of leading consulting firms and financial systems in hyper ledger, it meets the demanding and diverse needs of the financial services industry globally combining with the Linux foundation's as it is core technology.

$\mathrm{MC}$ was the optimization based technique where by using chain management is done this approach provides lots of features such as it is very effective in stability. The approach has its own challenges also like while implementing actual mass customization it fails and user cannot apply it fully. Regarding the delivery of goods which an integral part of SCM the delivery deadline should meet but it doesn't work effectively in this area. In BRIGS approach the authors have constructed a methodology which is an integration of TOPSIS and fuzzy DEMATEL.As it an integrated approach it was effective for gripping uncertainty and ambiguity. Also it provides flexibility of input as they have constructed decision matrix. The ultimate method is fuzzy DEMOPSIS. Two subsets criteria are used to identify weight; it cannot deal with big numbers of decision makers which is the big challenge. In OIPT approach author discussed the risk management strategies where the detection of risk factors is done which adversely affect the security; it was also effective to deal with interruption risk. It has major challenges of recovery from disruption which is very high. In EBC approach author developed a scale for determining EBC benefits it provides better SCM operation but the major challenge is computer complexity which is very high[19-22].

Transaction data should be treated as independent one and cannot trust by third-party hands, where it can be vulnerable to theft and misuse. Instead, users should have full control over their data without imposing restrictions or compromising security on companies, and have liberty to provide encrypted transactions.

The work done by researchers is discussed in related work. In the different methodologies suggested by researchers the cost of ownership management is very high and it consumes more time. To handle the problem the proposed methodologies is discussed in next section.

\section{Blockchain Based Privacy Preservation Approach}

By using blockchain technology a scheme for information sharing is implemented combines the blockchain technology in Supply Chain Management. The user does not need to trust any third party and conscious data is always used. Each participant in a blockchain network can see the progress of goods in the entire supply chain ecosystem. A decentralized, peer-to-peer model allows for open transparent cooperation with each other, with no chance of cheating or deception. No one can delete or change this transaction without the consent of the other players in the chain. The decentralized platform makes all operations faster, safer and more transparent without third-party involvement. Blockchain solutions will reduce transportation and payment delays.

In the proposed approach the data is gathered from all levels in the chain the overall architecture is discussed in Fig. 1 in which the supply chain compromise of different segments where data rotate from service sector to capacity sector with the intermediately level of inventory and load line. The methodologies consist of three process which is data sanitization, data restoration and key generation process by using key only the authorize person will be able to retrieve data from the chain and for key generation a new FSA-SLnO algorithm is discussed. As shown in Fig. 1 secured data sharing is done in every block of chain and the key is optimally selected using algorithm. In this process supply chain process is divided into four segments like A, B, C and D. where the segments are nothing but the different services offered by inventory and cost associated with it with total product structure. After generation of sensitive fields the blocks are created in which it compromises of hash key in header and transaction information by adding that each block is created forming a chain. Using this chain secured information is shared among different level through optimal key generation using the algorithm mention in further section.

A supply chain is a process in which multiple stakeholders are involved including manufacturer, manager, delivery and vendor which is shown in Fig. 2. At level 1 when a blockchain is created from data creator to manager level which is an endless chain of records at that level using data sanitization process which is sent to the next level of sensitive fields sanitized and sensitive field data is hidden from the intermediate level. 


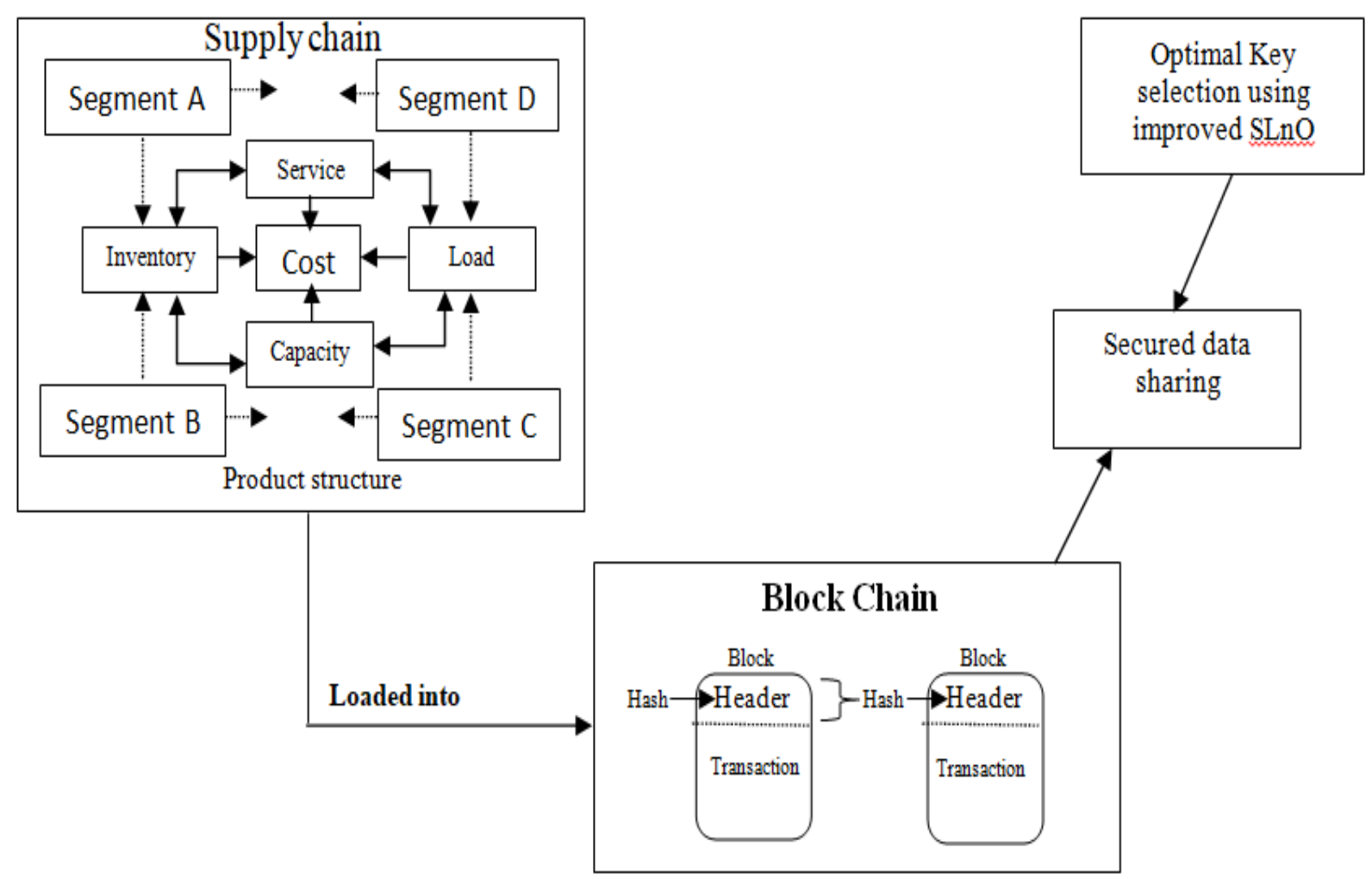

Fig. 1. Blockchain based privacy preservation approach

At the last level vendor having the optimal key is authorize user who can restore the data with data restoration process and access the original data.

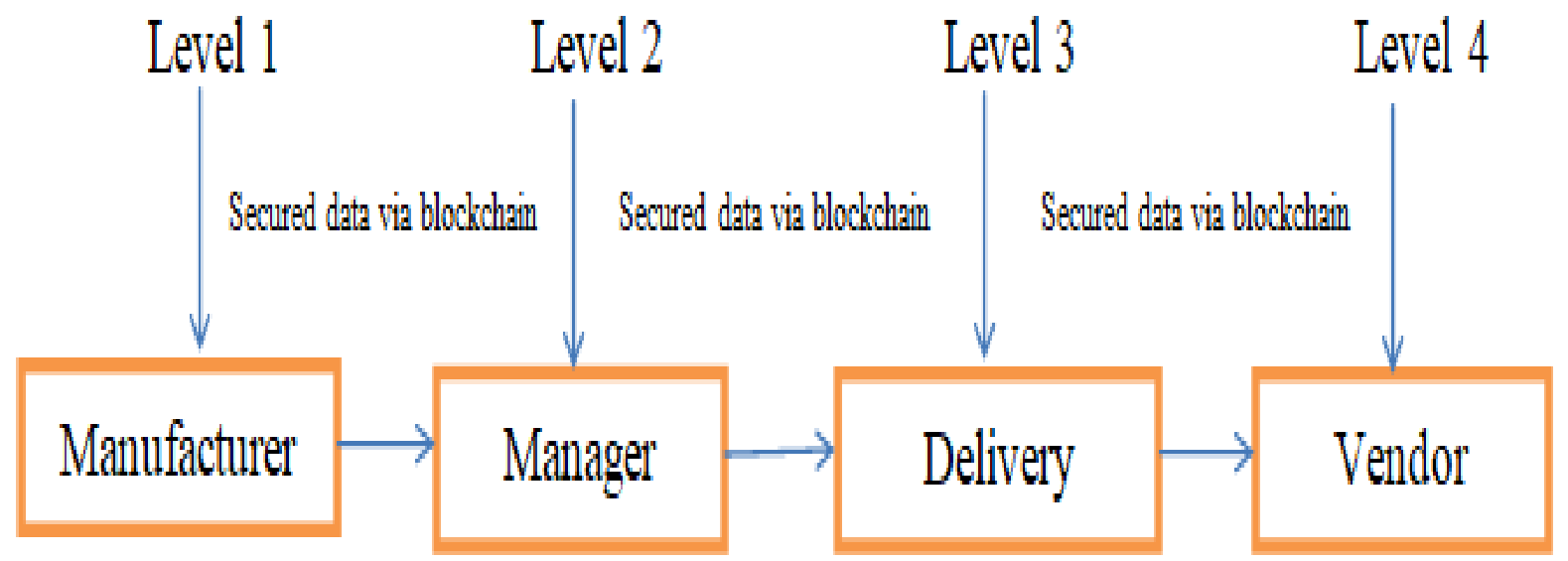

Fig. 2. Supply Chain Management Process

The count of block at each level is described in Table 1. In this for every level suppose for level 1, manufacture level the blocks generated are 88 where it is having more sensitive data as compared to rest of levels. In level 4 the count of blocks are 73 which is more as compared to level of manager and delivery as by using the key blocks is restored at this phase.

\begin{tabular}{|c|c|}
\hline Level & Count of blocks \\
\hline 1 & 88 \\
\hline 2 & 4 \\
\hline 3 & 2 \\
\hline 4 & 73 \\
\hline
\end{tabular}

Table 1. Count of Blocks 
For information sharing average time taken is shown in Table 2. In this the average time consumption for individual level for information sharing is given which is generated by considering the count of blocks for each level.

\begin{tabular}{|c|c|}
\hline Level & Average time (sec) \\
\hline 1 & 13.516 \\
\hline 2 & 27.418 \\
\hline 3 & 41.726 \\
\hline 4 & 16.32 \\
\hline
\end{tabular}

Table 2. Time consumed for information sharing

\section{Proposed Fittest Self Adaptive Sea Lion Optimization Algorithm (FSA-SLnO)}

The proposed algorithm is used for generating an optimal key so that when the data goes from intermediate levels by using the single key the authorized user should be able to use sensitive data.

The implemented FSA-SLnO methodology attains preservation of data which is shown in Eq. (1).

$$
\operatorname{Min} E=\max (E 1, E 2, E 3, E 4)
$$

Here,

E1, E2, E3 and E4 denote the related functions.

E1 denotes hiding failure rate in which $\max (\mathrm{E} 1)$ denotes worst of all iteration.

E2 denotes data preservation rate which is normalized.

E3 denotes false data generation which is normalized.

E4 denotes normalized modification degree.

The algorithm depicts the hunting behavior of sea lion which provide an optimal solution for key generation.

Step 1: Initialization of overall population and assigning index.

Step 2: Finding the fitness of overall population.

Step 3: If (spleader $<0.25$ )

For i.e. the first five best solutions of the fitness is updated using the Phase 1-Prey detection and tracking.

Step 4: In case if, then update the position of the solution using the exploration phase.

Step 5: Further, if(spleader $>=0.25$ ), then update the position of solution (first five solutions) using the exploitation of the standard Sea Lion optimization.

Step 6: For i.e. (next five solutions) Update the position of the solution with the new exploitation phase.

Using the algorithm the optimal key generation process is done through which only the authorize user will be able to access sensitive information.

\section{Result and Discussion}

\subsection{Simulation Procedure}

The proposed information sharing approach is implemented using MATLAB. The results acquired by using new approach is compared with the existing models like TOWPA,GOAGA, SACSA, FF and GWGA also the evolution is identified with the attacks and key sensitivity.

\subsection{Evolution on chosen cipher texts, Chosen-plaintext, Cipher text only, Known-plaintext attack}

This is an attack where it identifies the hidden secret key used for decryption. It is very crucial to have low value for cipher text. The generated evaluation is shown in Fig. 3 cipher texts of the presented work has the lowest value all over the varying cipher text and at $10 \%$, the work presented is $9.5 \%, 10.5 \%, 8.6 \%, 7.6 \%$ and 11.45 which is better than the existing models like TOWPA, GOAGA, SACSA, FF and GWGA. 


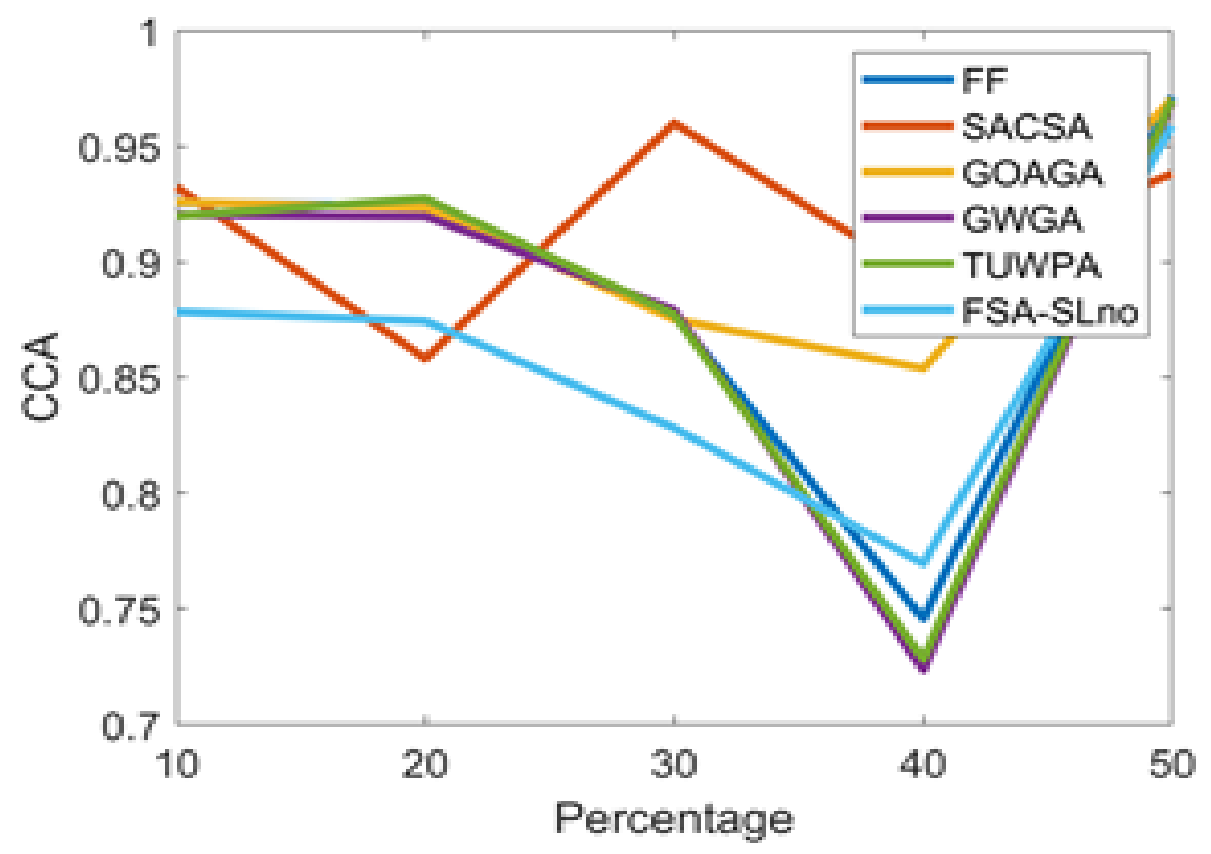

Fig. 3. Evolution on chosen cipher texts

Another attack Chosen-plaintext is an attack model of cryptanalysis that assumes that the attacker can obtain cipher texts for an arbitrary plaintext. The goal of this attack security of encryption scheme is reduced here. The evaluation shows all the variation of the cipher text and at $10 \%$ of the variation, the implemented work is $14.2 \%, 16.6 \%, 11.7 \%, 9.9 \%$ and $40 \%$ better than existing models like TOWPA, GOAGA, SACSA, FF and GWGA as shown in Fig. 4.

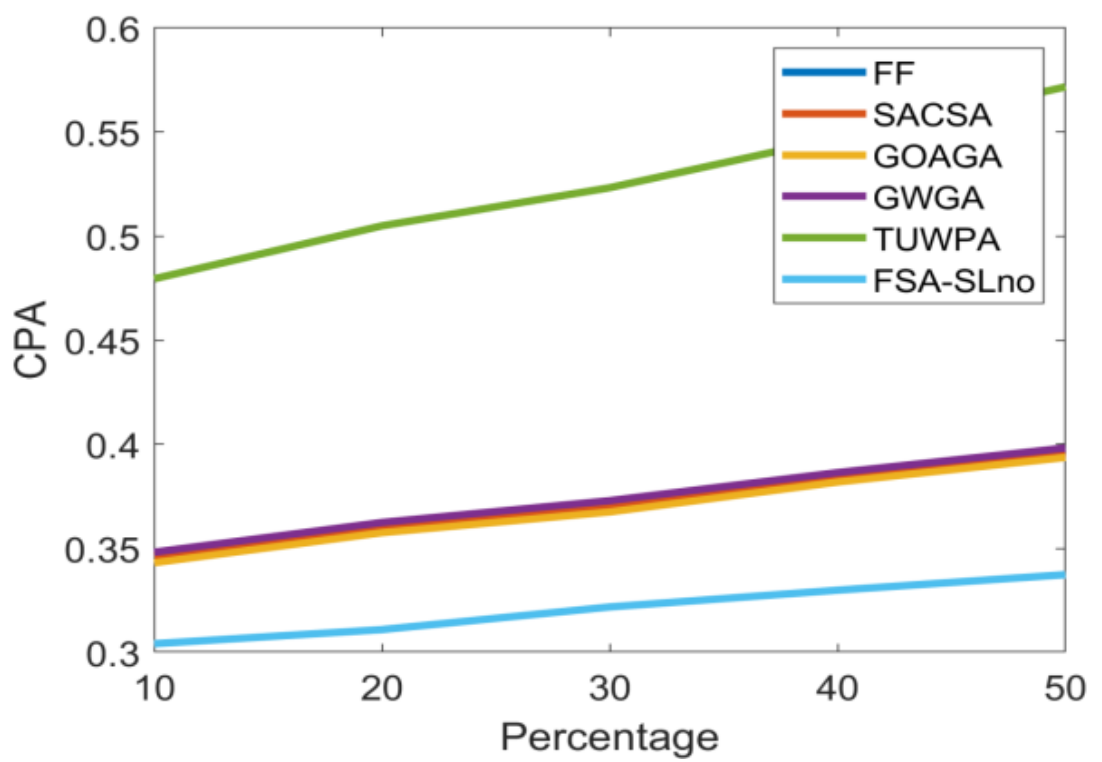

Fig. 4. Evolution on Chosen-plaintext

Out of remaining attacks known-plaintext attack is an attack model for cryptanalysis where the attacker has access to both the plaintext, and its encrypted version this attack is responsible for revealing code books and secret key information. The cryptanalysis of the implemented work for 8000 records, the presented work has the lowest value and it is $20 \%, 13.04 \%, 28.5 \%, 33.3 \%$ and $55.5 \%$ better than existing models like TOWPA, GOAGA, SACSA, FF and GWGA as shown in Fig. 5. 


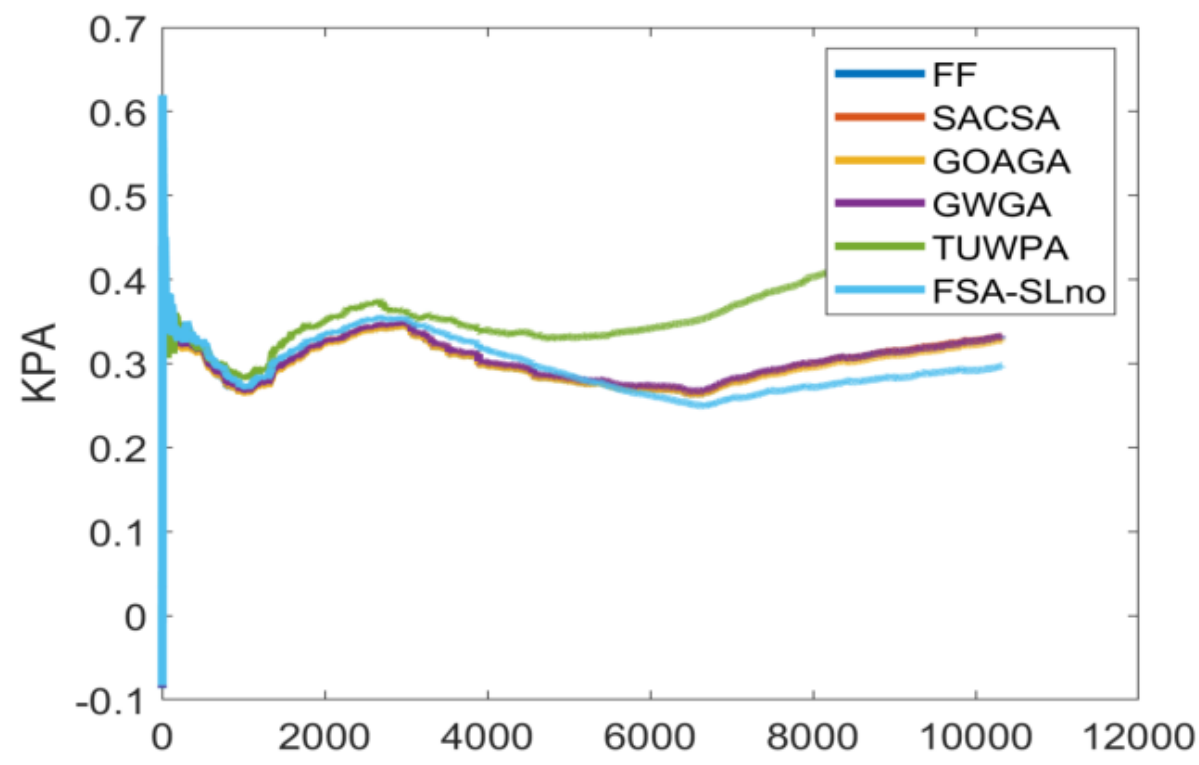

Fig. 5. Evolution on known-plaintext

Last attack which is Cipher text only, where the attacker is only perceived to have access to a set of cipher text. Although the attacker has no channels that allow access to plain text before encryption, in all practical cipher text only attacks the attacker still has some knowledge of the plaintext. After comparison it has the lowest value. Then for 10,000 records is $2.5 \%, 3.8 \%, 1.3 \%, 6.25 \%$ and $21.05 \%$ better than the existing models like TOWPA, GOAGA, SACSA, FF and GWGA as shown in Fig. 6.

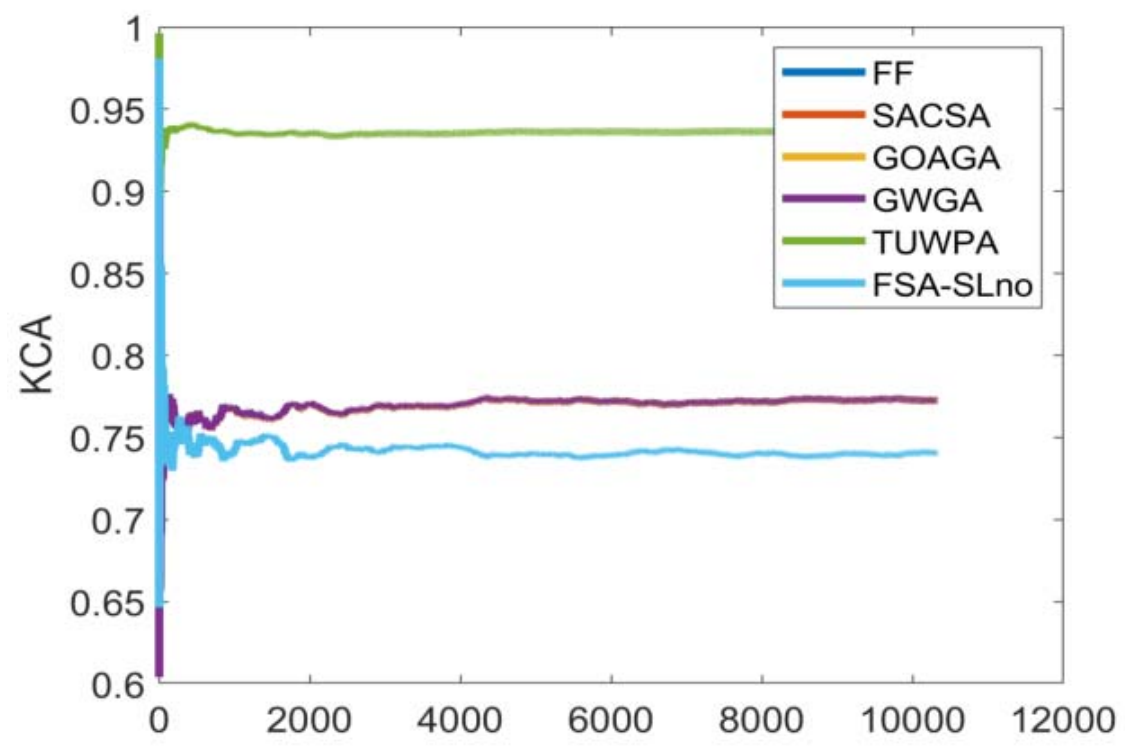

\section{Conclusion}

Fig. 6. Evolution on Cipher text-only

The current research work focusses on the privacy preservation in logistics area which is supply chain management where information sharing is crucial part and it has utmost importance while traversing from different level. Using blockchain technology the process of key generation is done and key is selected by implemented FSA-SLNO algorithm. From the results shown in section 5, it is clear that as compared to different attacks with different security models the proposed model always generates low value. B y using this technique only, the authenticated person will be able to retrieve data. 


\section{References}

[1] Kay Behnke, M. F. W. H. A. Janssen (2019): Boundary conditions for traceability in food supply chains using blockchain technology. International Journal of Information Management, Vol. 17, pp. 210-220.

[2] Petri Helo, A. H. M. Shamsuzzoha (2020): Real-time supply chain: A blockchain architecture for project deliveries. Robotics and Computer-Integrated Manufacturing, Vol.63.

[3] Tsan Ming Choi, Xin Wen, Xuting Sun, Sai-Ho Chung (2019): The mean variance approach for global supply chain risk analysis with air logistics in the blockchain technology era. Transportation Research Part E: Logistics and Transportation Review, Vol.127, pp.178191.

[4] Francesco Longo, Letizia Nicoletti, Antonio Padovano, Gianfranco d'Atri, Marco Forte (2019): Blockchain-enabled supply chain: An experimental study. Computers \& Industrial Engineering, Vol.136, pp. 57-69.

[5] Amit Karamchandani, Samir K. Srivastava, Rajiv K. Srivastavam (2019): Perception-based model for analyzing the impact of enterprise blockchain adoption on SCM in the Indian service industry. International Journal of Information Management.

[6] Andreas Kamilaris, Agusti Fonts, Francesc X. Prenafeta Boldú (2019): The rise of blockchain technology in agriculture and food supply chains. Trends in Food Science \& Technology, Vol.91, pp. 640-652.

[7] Ming Li, Jian Weng, Anjia Yang, Wei Lu,Yue Zhang, Lin Hou, Jia Nan Liu, Yang Xiang, Robert H. Deng (2018): CrowdBC: A Blockchain-based Decentralized Framework for Crowdsourcing. IEEE Transactions on Parallel and Distributed Systems.

[8] Magdalena Ramirez Peña, Alejandro J. Sánchez Sotano, Víctor Pérez Fernandez, Francisco J. Abad, Moises Batista (2019): Achieving a sustainable shipbuilding supply chain under I4.0 perspective. Journal of Cleaner Production, in communication.

[9] Raja Masadeh, Basel A. Mahafzah and Ahmad Sharieh (2019): Sea Lion Optimization Algorithm. International Journal of Advanced Computer Science and Applications(IJACSA), Vol. 10, No.5.

[10] M M Annie Alphonsa, N. Mohana Sundaram (2019): A reformed grasshopper optimization with genetic principle for securing medical data. Journal of Information Security and Applications, Vol. 47, pp. 410-420.

[11] Hokey Min (2019): Blockchain technology for enhancing supply chain resilience. Business Horizons, Vol.62, No.1, pp. 35-45.

[12] G. K. Shailaja, C. V. Guru Rao (2018): Robust and lossless data privacy preservation: optimal key based data sanitization. Evolutionary Intelligence.

[13] Geeta S. Navale, Suresh N. Mali (2018): A multi-analysis on privacy preservation of association rules using hybridized approach. Evolutionary Intelligence.

[14] MaozhuJin, Hua Wang, Qian Zhang, Yucheng Zeng (2018): Supply chain optimization based on chain management and mass customization. Information Systems and e-Business Management.

[15] Ediga Sathyanarayana Phalguna Krishna ,Thangavelu Arunkumar (2021): Hybrid Particle Swarm and Gray Wolf Optimization Algorithm for IoT Intrusion Detection System. International Journal of Intelligent Engineering and Systems, Vol.14, No.4.

[16] Avinash Samvedi, Vipul Jain, F. T. S. Chan, S. H. Chung (2016): Information system selection for a supply chain based on current trends: the BRIGS approach. Neural Computer \& Appllications.

[17] Scott Du Hadway, Steven Carnovale, Benjamin Hazen (2017): Understanding risk management for intentional supply chain disruptions: risk detection, risk mitigation, and risk recovery. Ann Oper Res.

[18] Amit Karamchandani, Samir K. Srivastava, Rajiv K. Srivastavam (2019): Perception-based model for analyzing the impact of enterprise blockchain adoption on SCM in the Indian service industry. International Journal of Information Management.

[19] M. Ganesh Karthik, M. B. Mukesh Krishnan (2021): Detecting Internet of Things Attacks Using Post Pruning Decision Tree-Synthetic Minority Over Sampling Technique. International Journal of Intelligent Engineering and Systems, Vol.14, No.4.

[20] Assunta Di Vaioa, Luisa Varriale (2019): Blockchain technology in supply chain management for sustainable performance: Evidence from the airport industry. International Journal of Information Management.

[21] Priti Lale, Dr.Rajesh Purohit (2020): Secured Exchange of Information in Supply Chain Management: A Literature Review. TEST Engineering and Management, ISSN: 0193-4120, pp. 8859-8862.

[22] Priti Lale, Dr. Manish Sharma (2020): Secured Information Sharing using Data Sanitization and restoration. International Journal of Advanced Trends in ComputerScience and Engineering, Vol. 9, No. 5.

[23] Andreas Kamilaris, Agusti Fonts, Francesc X. Prenafeta (2019): The rise of blockchain technology in agriculture and food supply chains. Trends in Food Science \& Technology, Vol. 91, pp. 640-652.

[24] Shuchih Ernest Chang, Yi Chian Chen, Ming Fang Lu (2019): Supply chain re-engineering using blockchain technology: A case of smart contract based tracking process. Technological Forecasting \& Social Change, No. 144, pp. 1-11.

[25] Scott Du Hadway Benjamin Hazen, Steven Carnovale (2018): Understanding risk management for intentional supply chain disruptions: risk detection, risk mitigation, and risk recovery. Ann Oper Res, DOI 10.1007/s10479-017-2452-0.

[26] Geeta S. Navale, Suresh N. Mali (2019): A multi analysis on privacy preservation of association rules using hybridized approach. Springer-Verlag GmbH Germany, https://doi.org/10.1007/s12065-019-00277-8.

\section{Authors Profile}

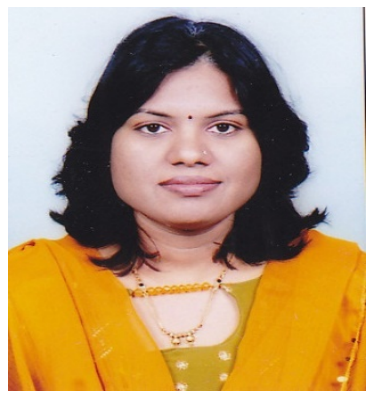

Priti R. Lale is a research scholar in Computer Science \& Engineering at Suresh Gyan Vihar University in Jaipur, India. She obtained her master's degree in Computer Science \& Engineering in 2014 from Sant Gadgebaba University, Amaravati, Maharashtra, India. Currently, she is working as an Assistant Professor in the Department of Information Technology, Mumbai Education Trust, Bhujbal Knowledge City, Institute of Engineering, Nashik, Maharashtra, India. She has a total of 16 years of teaching experience. Her research interests include Blockchain Technology, Database Management Systems, Big Data and the Internet of Things. 


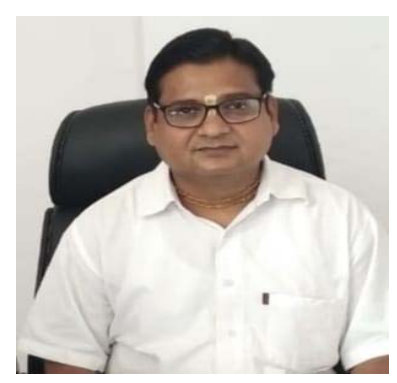

Dr. Mukesh Kumar Gupta did B.E in EIC from Rajasthan university Jaipur in 1995 and M.E in Electrical Engineering (Power System) from Rajasthan university in 2009. He had done Ph.D. in Electrical Engineering from Jagannath University Jaipur in 2014. His research area is Renewable energy and optimizing techniques. He is presently working as Professor and Associate Dean Research at Suresh Gyan Vihar University Jaipur (Rajasthan). He has published 65 papers and 11 patents and copyrights.

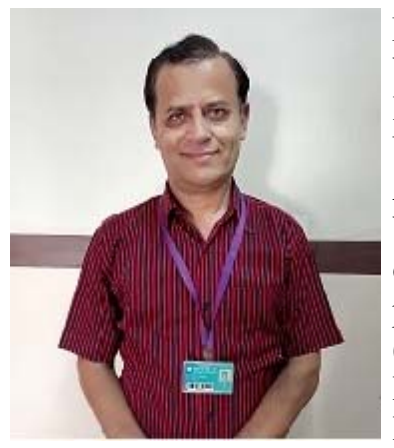

Dr. Manish Sharma is Ph.D. in Computer Science \& Engineering from Suresh Gyan Vihar University, Jaipur. He has done his M. Tech. (Computer Science) from Birla Institute of Technology, Mesra, Ranchi in 2000, and M.Sc. in Mathematics from University of Rajasthan, Jaipur in 1992. He is currently working as Director IQAC and Associate Professor in Department of Computer Engineering and IT in Suresh Gyan Vihar University. He has participated and presented many papers in various conferences and seminars of national/international repute. He has undertaken various projects like Market Gold (Developed in VB-6 and SQL-Server), Stock Gold (Developed in VB-6 and SQL-Server), Electricity Billing System in BIPS Info-Tech Private Ltd., Jaipur, Computer Integrated Home Security System (Developed in C++), Electronic controlling integrated in $\mathrm{C}$ language. 